\title{
Substance Index for Volume 68
}

Acetyl-2,4,10,17-tetrahydroxyheptadecylamine, $N$ -

Acetyl-3,5,11,18-tetrahydroxyoctadecyl-2-amine, $\mathrm{N}$ -

267

Acremolin B

Adipostatin A, B, C, D

Albaflavenol B

Allantopyrone A

Amauromine

Androprostamine A, B

Anidulafungin

Ansamycin analogues

Arbekacin, 4" -deoxy-4" -epiamino

Arbekacin, 6"-amino-6"-deoxy

Arcyriaflavin E

Ascosteroside C

Avellanin C

Bafilomycin L

Barceloneic acid C

BE-31405

Botryoisocoumarin A

Candicidin D

Carbamidocyclophane H, I, J, K, L

Carbonarin I, J

Caspofungin

Chandrananimycin E

Ciclopirox

Cinatrin D, E

Ciprofloxacin

Citreodiol

Citreodiol, epi-

Cladosporone A

Clonoamide

Coculnol

Cycloexpansamine A, B

Cystodienoic acid

Dandamycin

Daptomycin

Deoxaphomin, 20-oxo-

Deoxyspirobenzofuran

Deoxytetrodecamycin, 13-

Diastaphenazine

Dibekacin, 1-N-[(S)- $\omega$-amino-2-hydroxyalkyl $]$

derivative

Dibekacin, 5-deoxy

Doripenem

Emindole SC

Endophenazine A, glycosylated

Endophenazine B, glycosylated

Endophenazine C, glycosylated

Endophenazine D, glycosylated

Endophenazine E, glycosylated

Endophenazine F1, $N$-prenylated
Enniatin B2, Q

Epogymnolactam, (+)-

Fluconazole

Fosfomycin/colistin $\quad 551$

FR901459 511

Furanones $\quad 297$

Gentamicin B $\quad 421$

Goadsporin $\quad 594$

Graphiumin A, B, C, D, E, F, G, H 620

Gypsetin $\quad 638$

Herbimycin B, (15S)-15-hydroxy 476

Herbimycin B, 4,5-dihydro-(4S)-4-hydroxy 476

Hydropyrene $\quad 385$

Hydropyrenol 385

Hydroxy-10-methyldodec-2-en-1,4-olide, 4- 345

Hydroxy-10-methylundec-2-en-1,4-olide, 4- 345

Imipenem

2-(1'H-Indole-3'-carbonyl)-thiazoles $\quad 60$

Irciniastatin A

JBIR-140

K10-0216 KA, KB

Kalafungin, $5 R$-(N-acetyl-L-cysteinyl)-14Shydroxy-dihydro

Kanamycin A, 3'-deoxy

Lentztrehalose A, B, C

Leucinostatin I, II, III, IV, V 178

Levofloxacin

Liamocin A1, A2, B1, B2, C1, C2 642

Linezolid

Mangromicin C

MBJ-0086, -0087

Meropenem/clavulanate potassium $\quad 35$

Methoxyspirobenzofuran $\quad 351$

Methylbuthyl hydrodisulfide, 3- $\quad 414$

Micafungin 556

Milbemycin $A_{3}, 23$,24-didehydro-13 $\alpha$-hydroxy 354

Milbemycin $A_{3}, 24,30$-didehydro-13 $\alpha$-hydroxy 354

Misamycin $\quad 216$

Multirostratin A $\quad 23$

Neaumycin A, B, C 406

Nectchlorin A, B $\quad 47$

Nilotinib $\quad 469$

Novonestmycin A, B $\quad 185$

$\begin{array}{ll}\text { Odyverdiene A, B } & 385\end{array}$ 
Paradictyoarthrin A, B

Pazufloxacin

Penicillimide

Penipurdin A, B

Pentamycin

Phenylethyl hydrodisulfide

Phenylpyropene E, F

Phomenol

PM2409

Polymyxin B

Posaconazole

Pyripyropene A

Quinuclidine 1

Rebeccamycin

RK-270A, B, C

Rugulosin A, (+)-

Salinamide F

Sarkomycin analog

Sartorypyrone D

Scalbucillin A, B, C, D

Schisandrin

Schisandrin A, B, C

Schisandrol B

Schisantherin A

Sclerolizine

SF2446A2

Skyrin, (-)-R-

293

586

206

760

403

191

609

609

609

609

603

674

586
SMTP-52, -57, -58, -60, -61, -62

685

Spirobenzopyran derivative

351

641

271

Stachybotrin G

339

Staurosporine

594

Stemphol A, B

Streptazolin

Sydoxanthone C

501

40

703

Tolypoalbin

Trichodenol A, B

Trichoderamide A, B

409

409

628

757

373

385

Trienomycin B, E

271

UT-01309, -01320

76,485

Vancomycin

Vancomycin-sugar conjugates

302

Virgaricin B

633

Voriconazole

Xiakemycin A

Yanuthone K, L, M

201

Yanuthone X2

201

Zanthoxylumine A, B, C

289 